Supplement of Biogeosciences, 17, 2579-2591, 2020

https://doi.org/10.5194/bg-17-2579-2020-supplement

(C) Author(s) 2020. This work is distributed under

the Creative Commons Attribution 4.0 License.

(c) (1)

Supplement of

\title{
Reconstructing $\mathbf{N}_{2}$-fixing cyanobacterial blooms in the Baltic Sea beyond observations using 6- and 7-methylheptadecane in sediments as specific biomarkers
}

Jérôme Kaiser et al.

Correspondence to: Jérôme Kaiser (jerome.kaiser@io-warnemuende.de)

The copyright of individual parts of the supplement might differ from the CC BY 4.0 License. 
Table S1. Location of sediment trap, sediment cores, and monitoring stations.

\begin{tabular}{|c|c|c|c|c|}
\hline & Station & Lat. $\mathrm{N}$ & Long. E & Water depth $(\mathrm{m})$ \\
\hline \multirow{4}{*}{ 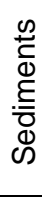 } & Core MSM51/20 & $57^{\circ} 59.866$ & $19^{\circ} 52.845$ & 198 \\
\hline & Core EMB1215/7 & $57^{\circ} 59.950$ & $19^{\circ} 52.950$ & 197 \\
\hline & Core POS435/10 & $62^{\circ} 52.160$ & $19^{\circ} 02.550$ & 214 \\
\hline & Sediment trap & $57^{\circ} 18.300$ & $20^{\circ} 00.460$ & 180 \\
\hline \multirow{14}{*}{ 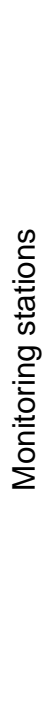 } & 32 & $57^{\circ} 58.500$ & $20^{\circ} 31.999$ & -- \\
\hline & $34 a$ & $57^{\circ} 58.000$ & $21^{\circ} 33.000$ & -- \\
\hline & BMP J1 & $57^{\circ} 19.200$ & $20^{\circ} 03.000$ & -- \\
\hline & BY15 & $57^{\circ} 19.200$ & $20^{\circ} 03.000$ & -- \\
\hline & BY15 W & $57^{\circ} 18.899$ & $19^{\circ} 54.600$ & -- \\
\hline & GB_12 & $57^{\circ} 11.640$ & $19^{\circ} 48.822$ & -- \\
\hline & GB_B13 & $57^{\circ} 26.652$ & $19^{\circ} 48.840$ & -- \\
\hline & GB_B14 & $57^{\circ} 11.658$ & $20^{\circ} 16.644$ & -- \\
\hline & TF0260 & $56^{\circ} 38.004$ & $19^{\circ} 34.998$ & -- \\
\hline & TF0270 & $57^{\circ} 51.900$ & $20^{\circ} 11.412$ & -- \\
\hline & TF0271 & $57^{\circ} 19.212$ & $20^{\circ} 03.018$ & -- \\
\hline & TF0271b & $57^{\circ} 18.348$ & $20^{\circ} 04.650$ & -- \\
\hline & TF0272 & $57^{\circ} 04.002$ & $19^{\circ} 49.986$ & -- \\
\hline & TF0286 & $58^{\circ} 00.000$ & $19^{\circ} 54.000$ & -- \\
\hline
\end{tabular}


Table S2. Core MSM51-2/20 age model based on an event stratigraphy.

\begin{tabular}{|r|l|r|l|}
\hline Depth $(\mathrm{cm})$ & Age & $\mathrm{SR}^{*}(\mathrm{~cm} / \mathrm{yr})$ & Event \\
\hline 0.00 & 2016 & 0.2 & Core recovery \\
\hline 2.75 & 2003 & 0.2 & Major Baltic Sea Inflow \\
\hline 9.00 & 1994 & 0.7 & Major Baltic Sea Inflow \\
\hline 12.75 & 1986 & 0.5 & Tchernobyl accident \\
\hline 16.90 & 1977 & 0.5 & Major Baltic Sea Inflow \\
\hline 22.00 & 1963 & 0.4 & start of atom waepons tests \\
\hline 25.00 & 1954 & 0.3 & maximum in atom waepons tests \\
\hline 31.00 & 1935 & 0.3 & start of PCB global production \\
\hline 35.00 & 1900 & 0.1 & rising use of Pb-containing petroleum \\
\hline 44.00 & 1860 & 0.2 & linear extrapolation \\
\hline
\end{tabular}

* Sedimentation Rate 
Table S3. Trap sediment material, monitoring, and satellite data from the Eastern Gotland Basin.

\begin{tabular}{|c|c|c|c|c|c|c|c|c|c|c|}
\hline \multicolumn{6}{|c|}{ Sediment trap } & \multicolumn{3}{|c|}{ Monitoring } & \multicolumn{2}{|c|}{ Satellite } \\
\hline Date & $7 \mathrm{Me}-\mathrm{C}_{17: 0}\left(\mu \mathrm{g} / \mathrm{m}^{2} /\right.$ day $)$ & $6 \mathrm{Me}-\mathrm{C}_{17: 0}\left(\mu \mathrm{g} / \mathrm{m}^{2} /\right.$ day $)$ & $6+7 \mathrm{Me}-\mathrm{C}_{17: 0}\left(\mu \mathrm{g} / \mathrm{m}^{2} / \mathrm{day}\right)$ & Aphanizomenon spp.* & Nodularia spp.* & Date & Aphanizomenon spp. $\left(\mathrm{mg} / \mathrm{m}^{3}\right)$ & Nodularia spp. $\left(\mathrm{mg} / \mathrm{m}^{3}\right)$ & Date & FCA \\
\hline $5 / 17 / 2010$ & 29.9 & 5.4 & 35.3 & 1 & & $5 / 6 / 2010$ & 3.1 & -- & 6/2/2010 & 0.0 \\
\hline $5 / 27 / 2010$ & 7.0 & 1.6 & 8.7 & 1 & & $5 / 17 / 2010$ & 3.9 & -- & $6 / 7 / 2010$ & 0.0 \\
\hline $6 / 21 / 2010$ & -- &.- & -- & -- & & $6 / 3 / 2010$ & 16.3 & -- & $6 / 12 / 2010$ & 0.0 \\
\hline $7 / 11 / 2010$ &.- &.- &.- & -- & & $7 / 1 / 2010$ & 46.2 & 9.7 & $6 / 17 / 2010$ & 0.0 \\
\hline $7 / 16 / 2010$ & 104.9 & 23.5 & 128.4 & 3 & 2 & $7 / 22 / 2010$ & 42.1 & 95.3 & $6 / 22 / 2010$ & 0.0 \\
\hline $7 / 26 / 2010$ & 2.9 & 0.6 & 3.4 & 3 & 2 & $8 / 19 / 2010$ & 25.7 & 43.2 & $6 / 27 / 2010$ & 0.0 \\
\hline $8 / 5 / 2010$ & 7.8 & 1.9 & 9.7 & 2 & 1 & $9 / 16 / 2010$ & 6.6 & -- & $7 / 2 / 2010$ & 0.2 \\
\hline $8 / 15 / 2010$ & 164.9 & 33.3 & 198.2 & 2 & 2 & $10 / 4 / 2010$ & 36.0 & -- & $7 / 7 / 2010$ & 5.3 \\
\hline 9/4/2010 & 43.4 & 10.0 & 53.4 & 2 & 1 & $11 / 11 / 2010$ & 4.6 & -- & 7/12/2010 & 38.4 \\
\hline 9/24/2010 & -- &.- & -- & 1 & & $11 / 16 / 2010$ & 14.2 & -- & $7 / 17 / 2010$ & 21.5 \\
\hline $10 / 4 / 2010$ & 38.3 & 9.7 & 48.1 & 3 & 1 & 1/13/2011 & -- & -- & $7 / 22 / 2010$ & 21.4 \\
\hline $10 / 24 / 2010$ & -- & -- & -- & 2 & & 2/7/2011 & 0.4 & -- & 7/27/2010 & 15.8 \\
\hline $11 / 25 / 2010$ & -- & - & -- & -- & & $3 / 12 / 2011$ & 0.1 & -- & $8 / 1 / 2010$ & 0.6 \\
\hline $12 / 1 / 2010$ & -- & -- & -- & -- & & $3 / 26 / 2011$ & - & -- & $8 / 6 / 2010$ & 0.2 \\
\hline $12 / 7 / 2010$ & -- & -- & -- & 1 & & $4 / 10 / 2011$ & -- & -- & $8 / 11 / 2010$ & 0.0 \\
\hline $12 / 13 / 2010$ &.- & -- & -.. & 1 & & $5 / 14 / 2011$ & 19.8 & -- & $8 / 16 / 2010$ & 0.6 \\
\hline $12 / 25 / 2010$ &.- & -- &.- & 2 & & & & & $8 / 21 / 2010$ & 0.0 \\
\hline 1/9/2011 &.- & -- & -- & 1 & & & & & $8 / 26 / 2010$ & 1.1 \\
\hline & & & & & & & & & $8 / 30 / 2010$ & 0.0 \\
\hline
\end{tabular}

* 1: present; 2: abundant; 3: highly abundant 
Table S4. Data from core MSM51-2/20, monitoring stations (summer and annual means), and satellite imagery (summer) for the period $1983-2016$.

\begin{tabular}{|c|c|c|c|c|c|c|c|c|}
\hline \multirow[b]{2}{*}{ Year } & \multirow[b]{2}{*}{ 7Me-C $17: 0(\mu \mathrm{g} / \mathrm{gTOC})$} & \multirow[b]{2}{*}{$6 \mathrm{Me}^{-\mathrm{C}_{17: 0}(\mu \mathrm{g} / \mathrm{gTOC})}$} & \multirow[b]{2}{*}{$6+7 \mathrm{Me}-\mathrm{C}_{17: 0}(\mu \mathrm{g} / \mathrm{gTOC})$} & \multicolumn{2}{|c|}{ Summer (July-August) } & \multicolumn{2}{|c|}{ Annual } & \multirow[b]{2}{*}{ FCA } \\
\hline & & & & Aphanizomenon spp. $\left(\mathrm{mg} / \mathrm{m}^{3}\right)$ & Nodularia spp. $\left(\mathrm{mg} / \mathrm{m}^{3}\right)$ & Aphanizomenon spp. $\left(\mathrm{mg} / \mathrm{m}^{3}\right)$ & Nodularia spp. $\left(\mathrm{mg} / \mathrm{m}^{3}\right)$ & \\
\hline 2016 & -- & & -- & 33.6 & 121.6 & 15.0 & 24.4 & 14.3 \\
\hline 2015 & 0.96 & 0.23 & 1.19 & 120.5 & 66.0 & 51.6 & 17.1 & 19.2 \\
\hline 2014 & -- & -- & -- & 75.6 & 161.6 & 21.0 & 38.5 & 43.5 \\
\hline 2013 & -- & -- & -- & 266.0 & 37.1 & 97.3 & 10.3 & 6.0 \\
\hline 2012 & 1.69 & 0.29 & 1.98 & 40.1 & 38.6 & 26.1 & 23.1 & 11.0 \\
\hline 2011 & -- & -- & -- & 66.8 & 89.0 & 25.3 & 28.0 & 18.6 \\
\hline 2010 & 1.05 & 0.18 & 1.22 & 36.3 & 57.3 & 15.0 & 16.0 & 11.0 \\
\hline 2009 & -- & -- & -- & 186.3 & 48.9 & 59.9 & 14.3 & 5.7 \\
\hline 2008 & 1.06 & 0.20 & 1.26 & 85.8 & 149.1 & 46.3 & 41.5 & 47.2 \\
\hline 2007 & -- & -- & -- & 122.5 & 44.5 & 32.3 & 8.9 & 7.2 \\
\hline 2006 & -- & -- & -- & 12.2 & 7.3 & 7.2 & 1.5 & 14.0 \\
\hline 2005 & 1.03 & 0.23 & 1.26 & 145.0 & 335.4 & 48.7 & 84.6 & 44.8 \\
\hline 2004 & -- & -- & -- & 73.2 & 256.8 & 33.6 & 77.5 & 9.7 \\
\hline 2003 & 0.64 & 0.12 & 0.76 & 34.6 & 58.8 & 14.6 & 16.4 & 41.9 \\
\hline 2002 & 0.29 & 0.05 & 0.34 & 109.6 & 177.6 & 38.6 & 49.4 & 17.6 \\
\hline 2001 & 0.20 & 0.05 & 0.25 & 121.1 & 39.3 & 39.8 & 10.9 & 4.7 \\
\hline 2000 & 0.46 & 0.07 & 0.52 & 25.2 & 113.6 & 12.3 & 28.4 & 25.7 \\
\hline 1999 & 1.34 & 0.25 & 1.58 & 56.5 & 192.8 & 28.4 & 48.3 & 37.3 \\
\hline 1998 & 0.63 & 0.11 & 0.74 & 52.0 & 41.4 & 26.4 & 10.7 & 7.7 \\
\hline 1997 & 0.38 & 0.07 & 0.45 & 239.8 & 56.0 & 75.2 & 14.2 & 11.2 \\
\hline 1996 & 0.02 & n.d. & 0.02 & 47.1 & 69.7 & 39.4 & 27.8 & 3.1 \\
\hline 1995 & 0.05 & n.d. & 0.05 & 104.1 & 60.8 & 39.5 & 14.3 & 5.1 \\
\hline 1994 & 0.08 & n.d. & 0.08 & 319.1 & 228.4 & 97.7 & 65.3 & 24.4 \\
\hline 1993 & 0.22 & 0.06 & 0.28 & 141.6 & 84.0 & 47.9 & 25.1 & 6.7 \\
\hline 1992 & 0.40 & 0.09 & 0.50 & 69.3 & 2148.8 & 20.4 & 538.0 & 17.9 \\
\hline 1991 & 0.45 & 0.11 & 0.56 & 283.8 & 87.3 & 121.6 & 37.4 & 19.6 \\
\hline 1990 & 0.53 & 0.12 & 0.65 & -- & -- & 33.9 & 7.5 & 1.6 \\
\hline 1989 & 1.12 & 0.21 & 1.33 & -- & -- & 7.1 & 0.2 & 2.8 \\
\hline 1988 & 1.23 & 0.22 & 1.44 & 70.5 & 19.5 & 6.9 & 0.0 & 3.3 \\
\hline
\end{tabular}




\begin{tabular}{|c|c|c|c|c|c|c|c|c|}
\hline 1987 & 1.44 & 0.29 & 1.73 & 24.4 & 19.1 & 6.2 & 24.3 & 0.3 \\
\hline 1986 & 0.88 & 0.16 & 1.04 & 41.2 & 3097.9 & 12.0 & 886.2 & -- \\
\hline 1985 & 0.99 & 0.18 & 1.18 & 105.3 & 203.3 & 45.2 & 110.5 & -- \\
\hline 1984 & 0.13 & n.d. & 0.13 & 46.3 & 16.3 & 17.5 & 5.4 & -- \\
\hline 1983 & 0.15 & n.d. & 0.15 & 117.0 & 0.0 & 45.7 & 0.0 & -- \\
\hline
\end{tabular}


Table S5. Core MSM51-2/20 (7Me-C17:0, 6Me-C17:0, 6+7Me-C 17:0, and TOC), AMO (Enfield et al., 2001), NAO (Hurrell, 1995; Jones et al., 1997), HadISST1 (Rayner et al., 2003), and annual riverine P input (Gustafsson et al., 2012) data since 1860.

\begin{tabular}{|c|c|c|c|c|c|c|c|c|c|}
\hline Depth $(\mathrm{cm})$ & Year & $7 \mathrm{Me}-\mathrm{C}_{17: 0}(\mu \mathrm{g} / \mathrm{gTOC})$ & $6 \mathrm{Me}-\mathrm{C}_{17: 0}(\mu \mathrm{g} / \mathrm{gTOC})$ & $6+7 \mathrm{Me}-\mathrm{C}_{17: 0}(\mu \mathrm{g} / \mathrm{gTOC})$ & TOC $(\%)$ & AMO & winter (DJFM) NAO & summer $(\mathrm{J}-\mathrm{A}) \operatorname{HadISST1}\left({ }^{\circ} \mathrm{C}\right)$ & annual riverine $\mathrm{P}$ input (ktons) \\
\hline 0.25 & 2015 & 0.96 & 0.23 & 1.19 & 15.9 & 0.10 & 8.16 & -- & -- \\
\hline-- & 2014 & -- & -- & -- & -- & 0.09 & 8.21 & -- & -- \\
\hline-- & 2013 & -- & -- & -- & -- & 0.15 & -2.33 & -- & - \\
\hline 0.75 & 2012 & 1.69 & 0.29 & 1.98 & 14.6 & 0.20 & 8.23 & -- & -- \\
\hline-- & 2011 & -- & -- & -- & -- & 0.09 & -3.65 & -- & -- \\
\hline 1.25 & 2010 & 1.05 & 0.18 & 1.22 & 15.2 & 0.34 & -10.15 & -- & - \\
\hline-- & 2009 & -- & -- & -- & -- & 0.02 & -1.25 & -- & - \\
\hline 1.75 & 2008 & 1.06 & 0.20 & 1.26 & 14.4 & 0.12 & 5.47 & 17.6 & - \\
\hline-- & 2007 & -- & -- & -- & -- & 0.13 & 7.30 & 16.6 & -- \\
\hline-- & 2006 & -- & -- & -- & -- & 0.25 & -0.79 & 18.7 & 24.8 \\
\hline 2.25 & 2005 & 1.03 & 0.23 & 1.26 & 16.0 & 0.28 & -0.45 & 17.4 & 28.2 \\
\hline-- & 2004 & -- & -- & -- & -- & 0.19 & -0.81 & 17.1 & 30.6 \\
\hline 2.75 & 2003 & 0.64 & 0.12 & 0.76 & 14.6 & 0.22 & 1.59 & 18.0 & 24.5 \\
\hline 3.50 & 2002 & 0.29 & 0.05 & 0.34 & 11.3 & 0.05 & 3.16 & 18.3 & 32.0 \\
\hline 4.25 & 2001 & 0.20 & 0.05 & 0.25 & 10.9 & 0.10 & -2.00 & 18.0 & 35.1 \\
\hline 4.75 & 2000 & 0.46 & 0.07 & 0.52 & 10.8 & 0.01 & 7.39 & 16.0 & 36.0 \\
\hline 5.50 & 1999 & 1.34 & 0.25 & 1.58 & 10.6 & 0.10 & 3.93 & 18.0 & 37.4 \\
\hline 6.25 & 1998 & 0.63 & 0.11 & 0.74 & 11.3 & 0.36 & 3.20 & 15.5 & 42.5 \\
\hline 7.00 & 1997 & 0.38 & 0.07 & 0.45 & 10.6 & 0.04 & 0.70 & 18.6 & 34.6 \\
\hline 7.75 & 1996 & 0.02 & n.d. & 0.02 & 12.1 & -0.07 & -9.29 & 15.9 & 32.2 \\
\hline 8.25 & 1995 & 0.05 & n.d. & 0.05 & 12.5 & 0.12 & 9.75 & 16.4 & 40.3 \\
\hline 8.75 & 1994 & 0.08 & n.d. & 0.08 & 9.6 & -0.19 & 7.20 & 17.8 & 39.7 \\
\hline 9.25 & 1993 & 0.22 & 0.06 & 0.28 & 9.2 & -0.23 & 5.70 & 15.4 & 33.5 \\
\hline 9.75 & 1992 & 0.40 & 0.09 & 0.50 & 11.1 & -0.23 & 6.72 & 16.4 & 32.8 \\
\hline 10.25 & 1991 & 0.45 & 0.11 & 0.56 & 11.7 & -0.15 & 0.82 & 16.8 & 32.9 \\
\hline 10.75 & 1990 & 0.53 & 0.12 & 0.65 & 11.7 & -0.05 & 9.49 & 16.3 & 35.6 \\
\hline 11.25 & 1989 & 1.12 & 0.21 & 1.33 & 9.9 & -0.10 & 11.44 & 16.6 & 34.9 \\
\hline 11.75 & 1988 & 1.23 & 0.22 & 1.44 & 9.6 & -0.02 & 0.39 & 16.9 & 42.2 \\
\hline 12.25 & 1987 & 1.44 & 0.29 & 1.73 & 9.8 & 0.05 & 1.35 & 13.5 & 42.2 \\
\hline
\end{tabular}




\begin{tabular}{|c|c|c|c|c|c|c|c|c|c|}
\hline 12.75 & 1986 & 0.88 & 0.16 & 1.04 & 10.6 & -0.29 & -0.13 & 15.9 & 38.8 \\
\hline 13.25 & 1985 & 0.99 & 0.18 & 1.18 & 10.7 & -0.28 & -1.52 & 15.6 & 41.4 \\
\hline 13.75 & 1984 & 0.13 & n.d. & 0.13 & 9.6 & -0.22 & 2.97 & 16.6 & 36.8 \\
\hline 14.25 & 1983 & 0.15 & n.d. & 0.15 & 10.4 & -0.09 & 8.00 & 16.2 & 37.6 \\
\hline 14.75 & 1982 & 0.13 & n.d. & 0.13 & 9.6 & -0.23 & 0.99 & 16.6 & 40.4 \\
\hline 15.25 & 1981 & 0.08 & n.d. & 0.15 & 8.9 & -0.09 & 3.60 & 15.1 & 48.4 \\
\hline-- & 1980 & -- & -- & -- & -- & -0.03 & 0.29 & 16.1 & 45.2 \\
\hline 15.75 & 1979 & 0.16 & n.d. & 0.16 & 7.6 & -0.13 & -5.38 & 15.4 & 40.0 \\
\hline 16.25 & 1978 & 0.08 & n.d. & 0.08 & 8.3 & -0.19 & 1.32 & 15.1 & 38.4 \\
\hline 16.75 & 1977 & 0.06 & n.d. & 0.06 & 8.9 & -0.20 & -4.38 & 14.8 & 38.7 \\
\hline 17.25 & 1976 & 0.06 & n.d. & 0.06 & 8.7 & -0.38 & 2.34 & 15.8 & 25.5 \\
\hline 17.75 & 1975 & 0.30 & 0.11 & 0.41 & 10.2 & -0.31 & 4.63 & 17.1 & 34.2 \\
\hline-- & 1974 & -- & -- & -- & -- & -0.43 & 1.97 & 15.7 & 36.9 \\
\hline 18.25 & 1973 & 0.68 & 0.15 & 0.83 & 8.4 & -0.23 & 5.74 & 17.8 & 25.9 \\
\hline 18.75 & 1972 & 0.47 & 0.07 & 0.54 & 8.5 & -0.37 & 0.30 & 17.0 & 27.2 \\
\hline 19.25 & 1971 & 0.44 & n.d. & 0.38 & 8.2 & -0.32 & -2.56 & 15.2 & 28.9 \\
\hline-- & 1970 & -- & -- & -- & -- & -0.12 & -2.10 & 15.6 & 33.0 \\
\hline 19.75 & 1969 & 0.51 & 0.09 & 0.60 & 9.0 & 0.00 & -8.34 & 17.3 & 24.4 \\
\hline 20.25 & 1968 & 0.18 & n.d. & 0.18 & 8.4 & -0.18 & -0.07 & 16.3 & 30.8 \\
\hline -- & 1967 & -- & -- & -- & -- & -0.11 & 6.42 & 16.1 & 34.7 \\
\hline 20.75 & 1966 & 0.10 & n.d. & 0.10 & 8.6 & -0.01 & 0.90 & 15.7 & 31.7 \\
\hline 21.25 & 1965 & 0.20 & n.d. & 0.20 & 7.9 & -0.17 & -4.03 & 14.1 & 26.3 \\
\hline 21.75 & 1964 & 0.21 & n.d. & 0.21 & 8.1 & -0.11 & -3.06 & 14.7 & 20.9 \\
\hline -- & 1963 & -- & -- & -- & -- & -0.01 & -3.86 & 15.7 & 22.5 \\
\hline 22.25 & 1962 & 0.14 & n.d. & 0.14 & 7.6 & 0.06 & -2.29 & 13.9 & 30.9 \\
\hline 22.75 & 1961 & 0.60 & 0.14 & 0.74 & 9.8 & 0.09 & 8.06 & 15.1 & 24.3 \\
\hline 23.25 & 1960 & 0.82 & 0.12 & 0.94 & 9.4 & 0.23 & -0.59 & 15.6 & 22.9 \\
\hline 23.75 & 1959 & 0.26 & n.d. & 0.26 & 6.8 & 0.04 & 1.43 & 16.9 & 24.9 \\
\hline 24.25 & 1958 & 0.31 & n.d. & 0.31 & 6.5 & 0.21 & -1.06 & 14.6 & 27.0 \\
\hline 24.75 & 1957 & 0.20 & n.d. & 0.20 & 7.0 & 0.03 & 6.60 & 15.4 & 23.2 \\
\hline 25.25 & 1956 & 0.11 & n.d. & 0.11 & 4.9 & -0.03 & -3.20 & 14.8 & 21.1 \\
\hline 25.75 & 1955 & 1.00 & 0.24 & 1.24 & 3.0 & 0.18 & -4.84 & 16.4 & 20.7 \\
\hline-- & 1954 & -- & - & -- & -- & 0.03 & 0.53 & 15.7 & 17.5 \\
\hline
\end{tabular}




\begin{tabular}{|c|c|c|c|c|c|c|c|c|c|}
\hline 26.25 & 1953 & 0.71 & 0.15 & 0.86 & 3.3 & 0.26 & 1.46 & 16.4 & 18.9 \\
\hline-- & 1952 & -- & -- & -- & -- & 0.29 & 2.47 & 15.5 & 16.2 \\
\hline 26.75 & 1951 & 0.48 & n.d. & 0.48 & 3.3 & 0.20 & -2.14 & 15.3 & 16.9 \\
\hline-- & 1950 & -- & -- & -- & -- & -0.02 & 4.79 & 16.9 & 16.5 \\
\hline 27.25 & 1949 & 0.41 & n.d. & 0.41 & 2.6 & 0.09 & 5.64 & 15.8 & 13.8 \\
\hline -- & 1948 & -- & -- & -- & -- & 0.01 & 4.95 & 15.3 & 12.7 \\
\hline 27.75 & 1947 & 0.35 & n.d. & 0.35 & 2.7 & -0.09 & -4.27 & 16.4 & 10.5 \\
\hline-- & 1946 & -- & -- & -- & -- & 0.01 & 1.76 & 16.0 & 11.9 \\
\hline 28.25 & 1945 & 0.41 & n.d. & 0.41 & 3.1 & 0.21 & 5.32 & 16.7 & 13.0 \\
\hline 28.75 & 1944 & 0.52 & 0.10 & 0.62 & 3.1 & 0.34 & 0.83 & 16.1 & 12.4 \\
\hline-- & 1943 & -- & -- & -- & -- & 0.03 & 5.45 & 15.7 & 10.0 \\
\hline 29.25 & 1942 & 0.57 & 0.13 & 0.70 & 3.0 & 0.18 & -1.83 & 14.9 & 9.3 \\
\hline-- & 1941 & -- & -- & -- & -- & 0.17 & -3.75 & 14.7 & 11.5 \\
\hline 29.75 & 1940 & 0.71 & 0.17 & 0.88 & 3.0 & -0.03 & -4.99 & 15.7 & 10.4 \\
\hline-- & 1939 & -- & -- & -- & -- & 0.11 & 1.80 & 17.5 & 10.7 \\
\hline-- & 1938 & -- & -- & -- & -- & 0.24 & 5.28 & 17.2 & 10.6 \\
\hline 30.50 & 1937 & 0.95 & 0.24 & 1.19 & 2.5 & 0.29 & 4.23 & 18.8 & 10.3 \\
\hline-- & 1936 & -- & -- & -- & -- & 0.15 & -6.12 & 17.8 & 12.2 \\
\hline-- & 1935 & -- & -- & -- & -- & 0.02 & 3.91 & 16.3 & 11.8 \\
\hline -- & 1934 & -- & -- & -- & -- & -0.01 & 2.22 & 16.9 & 10.3 \\
\hline-- & 1933 & -- & -- & -- & -- & 0.19 & 2.08 & 16.6 & 10.1 \\
\hline-- & 1932 & -- & -- & -- & -- & 0.23 & -0.64 & 17.7 & 12.0 \\
\hline 31.50 & 1931 & 0.87 & 0.15 & 1.02 & 2.5 & 0.18 & 0.23 & 14.8 & 13.5 \\
\hline-- & 1930 & -- & -- & -- & -- & 0.01 & 4.01 & 16.8 & 11.8 \\
\hline-- & 1929 & -- & -- & -- & -- & -0.11 & -1.03 & 14.2 & 11.4 \\
\hline -- & 1928 & -- & -- & -- & -- & -0.01 & 2.48 & 13.9 & 12.0 \\
\hline-- & 1927 & -- & -- & -- & -- & 0.11 & 5.31 & 17.1 & 13.2 \\
\hline-- & 1926 & -- & - & -- & -- & 0.08 & 3.38 & 16.8 & 12.5 \\
\hline-- & 1925 & -- & -- & -- & -- & -0.16 & 8.04 & 16.5 & 11.0 \\
\hline-- & 1924 & -- & -- & -- & -- & -0.15 & -1.39 & 15.8 & 14.0 \\
\hline-- & 1923 & -- & - & -- & -- & -0.33 & 5.98 & 14.7 & 11.5 \\
\hline 32.50 & 1922 & 0.39 & n.d. & 0.39 & 2.5 & -0.32 & 5.56 & 15.6 & 10.4 \\
\hline-- & 1921 & -- & -- & - & -- & -0.22 & 5.49 & 15.1 & 8.1 \\
\hline
\end{tabular}




\begin{tabular}{|c|c|c|c|c|c|c|c|c|c|}
\hline-- & 1920 & -- & -- & -- & -- & -0.34 & 10.17 & 15.7 & 11.7 \\
\hline-- & 1919 & -- & -- & -- & -- & -0.19 & 1.57 & 14.4 & 10.8 \\
\hline-- & 1918 & -- & -- & -- & -- & -0.26 & 0.96 & 15.7 & 10.9 \\
\hline-- & 1917 & -- & -- & -- & -- & -0.28 & -6.68 & 15.0 & 11.5 \\
\hline-- & 1916 & -- & -- & -- & -- & -0.08 & 1.36 & 14.6 & 11.4 \\
\hline -- & 1915 & -- & -- & -- & -- & 0.09 & 2.22 & 15.4 & 9.6 \\
\hline-- & 1914 & -- & -- & -- & -- & -0.29 & 5.98 & 18.2 & 9.9 \\
\hline 33.50 & 1913 & 0.16 & n.d. & 0.16 & 2.5 & -0.39 & 8.75 & 16.1 & 11.0 \\
\hline-- & 1912 & -- & -- & -- & -- & -0.23 & 4.16 & 16.9 & 11.1 \\
\hline-- & 1911 & -- & -- & -- & -- & -0.21 & 1.37 & 15.8 & 10.0 \\
\hline-- & 1910 & -- & - & -- & -- & -0.25 & 6.49 & 15.2 & 10.5 \\
\hline-- & 1909 & -- & -- & -- & -- & -0.14 & 0.66 & 14.7 & 10.0 \\
\hline-- & 1908 & -- & -- & -- & -- & -0.13 & 4.54 & 15.8 & 9.8 \\
\hline-- & 1907 & -- & -- & -- & -- & -0.23 & 5.24 & 13.3 & 10.4 \\
\hline-- & 1906 & -- & -- & -- & -- & -0.07 & 5.50 & 15.9 & 10.8 \\
\hline-- & 1905 & -- & -- & -- & -- & -0.20 & 7.01 & 16.6 & 11.1 \\
\hline 34.50 & 1904 & 0.09 & n.d. & 0.09 & 2.4 & -0.35 & 1.66 & 13.9 & 11.6 \\
\hline-- & 1903 & -- & -- & -- & -- & -0.19 & 11.46 & 14.4 & 12.1 \\
\hline-- & 1902 & -- & -- & -- & -- & -0.10 & -0.87 & 14.2 & 10.2 \\
\hline-- & 1901 & -- & -- & -- & - & 0.09 & 0.99 & 16.0 & 9.5 \\
\hline-- & 1900 & -- & -- & -- & -- & 0.10 & -4.97 & 15.6 & 9.2 \\
\hline-- & 1899 & -- & -- & -- & -- & 0.13 & 2.15 & 16.2 & 10.0 \\
\hline 35.50 & 1898 & 0.04 & n.d. & 0.04 & 2.4 & 0.08 & 2.89 & 15.1 & 9.4 \\
\hline-- & 1897 & -- & -- & -- & -- & 0.11 & 5.13 & 15.7 & 9.0 \\
\hline-- & 1896 & -- & -- & -- & -- & 0.11 & 3.51 & 16.9 & 9.2 \\
\hline-- & 1895 & -- & -- & -- & -- & -0.09 & -6.08 & 15.3 & 9.9 \\
\hline-- & 1894 & -- & -- & -- & -- & -0.24 & 8.33 & 15.6 & 9.2 \\
\hline 36.50 & 1893 & 0.08 & n.d. & 0.08 & 2.3 & 0.00 & -0.16 & 15.5 & 10.2 \\
\hline-- & 1892 & -- & -- & -- & -- & -0.09 & -1.65 & 15.0 & 10.1 \\
\hline-- & 1891 & -- & -- & -- & -- & 0.04 & -0.84 & 16.0 & 8.8 \\
\hline-- & 1890 & -- & -- & -- & -- & -0.14 & 5.75 & 15.3 & 8.7 \\
\hline 37.50 & 1889 & 0.04 & n.d. & 0.04 & 2.4 & 0.20 & 1.81 & 15.5 & 9.2 \\
\hline-- & 1888 & -- & -- & -- & $-{ }_{-}^{-}$ & 0.20 & -5.16 & 14.0 & 10.7 \\
\hline
\end{tabular}




\begin{tabular}{|c|c|c|c|c|c|c|c|c|c|}
\hline-- & 1887 & -- & -- & -- & -- & 0.12 & 3.20 & 15.1 & 9.4 \\
\hline --. & 1886 & -- & -- & -- & -- & 0.13 & -1.27 & 15.8 & 8.9 \\
\hline-- & 1885 & -- & -- & -- & -- & -0.02 & 0.17 & 15.8 & 9.0 \\
\hline 38.50 & 1884 & 0.04 & n.d. & 0.04 & 2.5 & -0.07 & 5.90 & 16.7 & 7.9 \\
\hline-- & 1883 & -- & -- & -- & - & -0.03 & 1.19 & 15.9 & 9.3 \\
\hline-- & 1882 & -- & -- & -- & -- & -0.02 & 12.65 & 16.7 & 9.1 \\
\hline-- & 1881 & -- & -- & -- & -- & 0.05 & -5.21 & 14.8 & 9.9 \\
\hline 39.50 & 1880 & 0.12 & n.d. & 0.12 & 2.5 & 0.07 & 2.98 & 15.8 & 8.1 \\
\hline-- & 1879 & -- & -- & -- & -- & 0.13 & -3.25 & 15.3 & 9.8 \\
\hline-- & 1878 & -- & -- & -- & -- & 0.46 & 5.53 & 15.7 & 8.9 \\
\hline-- & 1877 & -- & -- & -- & -- & 0.25 & 2.77 & 14.6 & 10.0 \\
\hline 40.50 & 1876 & 0.09 & n.d. & 0.09 & 2.5 & -0.02 & 1.64 & 15.5 & 8.5 \\
\hline-- & 1875 & -- & -- & -- & -- & 0.04 & -2.14 & 16.5 & 8.0 \\
\hline-- & 1874 & -- & -- & -- & -- & -0.01 & 7.11 & 15.3 & 8.8 \\
\hline-- & 1873 & -- & -- & -- & -- & 0.05 & 1.00 & 15.6 & 8.8 \\
\hline-- & 1872 & -- & -- & -- & -- & 0.10 & 0.22 & 16.3 & 8.4 \\
\hline 41.50 & 1871 & 0.12 & n.d. & 0.12 & 2.5 & 0.04 & 0.01 & 14.6 & 8.6 \\
\hline-- & 1870 & -- & -- & -- & -- & 0.03 & -4.42 & 15.5 & 7.9 \\
\hline-- & 1869 & -- & -- & -- & -- & 0.10 & 7.25 & -- & 9.3 \\
\hline-- & 1868 & -- & -- & -- & -- & 0.16 & 7.80 & -- & 7.9 \\
\hline 42.50 & 1867 & 0.21 & n.d. & 0.21 & 2.4 & 0.15 & 0.57 & -- & 9.2 \\
\hline-- & 1866 & -- & -- & -- & -- & 0.21 & 3.23 & -- & 9.0 \\
\hline-- & 1865 & -- & -- & -- & -- & 0.18 & -2.07 & -- & 8.4 \\
\hline-- & 1864 & -- & -- & -- & -- & 0.07 & 2.15 & - & 8.1 \\
\hline-- & 1863 & -- & -- & -- & -- & -0.11 & 9.02 & -- & 8.3 \\
\hline-- & 1862 & -- & -- & -- & -- & -0.19 & -3.86 & -- & 7.9 \\
\hline-- & 1861 & -- & -- & -- & -- & 0.19 & 0.16 & -- & 8.2 \\
\hline-- & 1860 & -- & - & -- & - & 0.09 & 1.63 & - & 8.6 \\
\hline
\end{tabular}


Table S6. Correlation coefficients ( $\mathrm{r}$ ) and $\mathrm{p}$ values (in italic). $\mathrm{r}$ values are in bold for $\mathrm{p}<0.05$.

\begin{tabular}{|l|r|r|r|r|r|}
\hline & AMO & winter NAO & summer HadISST & P input & $6+7 \mathrm{Me} \mathrm{C}_{17: 0}$ \\
\hline AMO & & 0.03 & 0.00 & 0.01 & 0.01 \\
\hline winter NAO & $\mathbf{- 0 . 2 5}$ & & 0.13 & 0.76 & 0.43 \\
\hline summer HadISST & $\mathbf{0 . 3 8}$ & 0.18 & & 0.01 & 0.04 \\
\hline P input & $-\mathbf{0 . 3 0}$ & -0.04 & $-\mathbf{0 . 3 0}$ & & 0.47 \\
\hline $6+7$ Me-C $17: 0$ & $\mathbf{0 . 3 0}$ & 0.10 & $\mathbf{0 . 2 5}$ & -0.09 & \\
\hline
\end{tabular}


Table S7. Core POS435/10 data.

\begin{tabular}{|c|c|c|c|c|}
\hline Depth $(\mathrm{cm})$ & Age (yr cal. BP) & $7 \mathrm{Me}-\mathrm{C}_{17: 0}(\mu \mathrm{g} / \mathrm{gTOC})$ & $6 \mathrm{Me}-\mathrm{C}_{17: 0}(\mu \mathrm{g} / \mathrm{gTOC})$ & $6+7 \mathrm{Me}-\mathrm{C}_{17: 0}(\mu \mathrm{g} / \mathrm{gTOC})$ \\
\hline 0.25 & -61.8 & 0.90 & 0.30 & 1.18 \\
\hline 32.5 & -40.9 & 0.22 & 0.04 & 0.27 \\
\hline 58.5 & -0.6 & 1.10 & 0.30 & 1.41 \\
\hline 85 & 50.0 & 0.24 & 0.03 & 0.27 \\
\hline 115 & 130.1 & 0.50 & 0.20 & 0.69 \\
\hline 145 & 210.1 & 1.19 & 0.19 & 1.38 \\
\hline 175 & 290.2 & 0.70 & 0.20 & 0.92 \\
\hline 205 & 370.3 & 1.96 & 0.35 & 2.31 \\
\hline 260 & 533.5 & 0.64 & 0.23 & 0.87 \\
\hline 310 & 717.0 & 0.20 & 0.04 & 0.24 \\
\hline 340 & 827.1 & 0.28 & 0.07 & 0.36 \\
\hline 410 & 1083.9 & 0.48 & 0.10 & 0.58 \\
\hline 465 & 1285.7 & 1.05 & 0.25 & 1.30 \\
\hline 500 & 1414.2 & 0.30 & 0.11 & 0.41 \\
\hline 520 & 1515.3 & 0.18 & 0.04 & 0.20 \\
\hline 540 & 1617.2 & 1.00 & 0.26 & 1.26 \\
\hline 560 & 1719.0 & 0.66 & 0.19 & 0.86 \\
\hline 580 & 1820.9 & 1.17 & 0.33 & 1.50 \\
\hline 600 & 1922.7 & 0.17 & 0.03 & 0.18 \\
\hline 610 & 1977.4 & 0.40 & 0.10 & 0.49 \\
\hline 620 & 2053.1 & 0.23 & 0.06 & 0.25 \\
\hline 640 & 2204.7 & 1.57 & 0.36 & 1.93 \\
\hline 650 & 2280.5 & 1.87 & 0.65 & 2.52 \\
\hline 660 & 2466.8 & 2.60 & 0.60 & 3.20 \\
\hline 670 & 2642.3 & 1.82 & 0.49 & 2.30 \\
\hline 680 & 2817.8 & 0.20 & 0.05 & 0.28 \\
\hline 707 & 3291.6 & 0.13 & 0.02 & 0.15 \\
\hline 731 & 3733.3 & 0.12 & 0.03 & 0.14 \\
\hline 739 & 3884.0 & 0.13 & 0.05 & 0.25 \\
\hline 747 & 4034.7 & 0.69 & 0.17 & 0.86 \\
\hline
\end{tabular}




\begin{tabular}{|r|r|r|r|r|}
\hline 759 & 4395.9 & 1.94 & 0.45 & 2.39 \\
\hline 771 & 4824.8 & 0.30 & 0.08 & 0.41 \\
\hline 783 & 5253.7 & 0.14 & 0.05 & 0.23 \\
\hline 791 & 5539.7 & 0.14 & 0.05 & 0.18 \\
\hline 803 & 5833.1 & 0.29 & 0.09 & 0.38 \\
\hline 811 & 5922.0 & 0.12 & 0.05 & 0.17 \\
\hline 817 & 5988.7 & 0.20 & 0.07 & 0.24 \\
\hline 833 & 6166.4 & 9.50 & 3.00 & 12.50 \\
\hline 839 & 6230.2 & 17.20 & 4.00 & 21.20 \\
\hline 847 & 6296.1 & 61.30 & 15.57 & 76.87 \\
\hline 863 & 6529.2 & 51.14 & 10.98 & 62.12 \\
\hline 871 & 6662.6 & 218.19 & 62.74 & 280.93 \\
\hline 879 & 6808.6 & 220.00 & 66.60 & 286.56 \\
\hline 893.5 & 7080.8 & 0.24 & 0.05 & 0.29 \\
\hline 905.5 & 7306.0 & 0.30 & 0.06 & 0.41 \\
\hline
\end{tabular}

